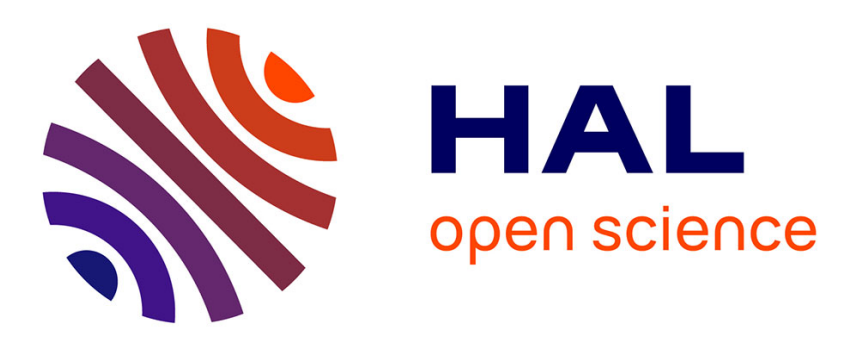

\title{
Extended Wannier ridge model for the electrons angular distribution
}

\author{
A. Kazansky, V. Ostrovsky
}

\section{To cite this version:}

A. Kazansky, V. Ostrovsky. Extended Wannier ridge model for the electrons angular distribution. Journal de Physique IV Proceedings, 1993, 03 (C6), pp.C6-159-C6-167. 10.1051/jp4:1993615 . jpa00251700

\section{HAL Id: jpa-00251700 https://hal.science/jpa-00251700}

Submitted on 1 Jan 1993

HAL is a multi-disciplinary open access archive for the deposit and dissemination of scientific research documents, whether they are published or not. The documents may come from teaching and research institutions in France or abroad, or from public or private research centers.
L'archive ouverte pluridisciplinaire HAL, est destinée au dépôt et à la diffusion de documents scientifiques de niveau recherche, publiés ou non, émanant des établissements d'enseignement et de recherche français ou étrangers, des laboratoires publics ou privés. 


\title{
Extended Wannier ridge model for the electrons angular distribution
}

\author{
A.K. KAZANSKY and V.N. OSTROVSKY \\ Institute of Physics, The University of St Petersburg, St Petersburg 198904, Russia
}

\begin{abstract}
The extended Wannier model describes the propagation of two-electron wave packet along the Wannier ridge from the reaction zone (where it is formed by $(\gamma, 2 e)$ or (e, 2e) process) to the zone of free electron motion. The angular correlation patterns are calculated and analyzed for ${ }^{1} \mathrm{~S}^{\mathrm{e}}$ and ${ }^{1} \mathrm{Po}^{\mathrm{s}}$ states of the two-electron continuum.
\end{abstract}

\section{Introduction}

The experimental measurements of the angular electron distributions for $(e, 2 e)$ and $(\gamma, 2 e)$ processes are feasible now in the near threshold region [1-3] where the well known Wannier [4] mechanism of the double electron escape is supposed to be dominant. For the theoretical description of the electron angular correlations we have proposed recently the extended Wannier ridge model described in detail elsewhere [5]. Below we outline its principal physical features.

(1) The two electron escape along the Wannier ridge $r_{1}=r_{2}$ is considered and the electron angular variables are separated from the radial variables $r_{1}, r_{2}$ (or, equivalently, from the hyperspherical radius $R$ $=\left(r_{1}{ }^{2}+r_{2}\right)^{1 / 2}$ and the hyperangle $\alpha_{h}=\operatorname{atan}\left(r_{1} / r_{2}\right)$. Here $r_{1}$ and $r_{2}$ are the electron radius-vectors relative to the atomic nucleus.

(2) The quadratic expansion of the Hamiltonian near the point $\theta_{12}=\pi$ is not employed in contradistinction to the standard $[4,6]$ Wannier approximation $\left(\theta_{12}\right.$ is the angle between the vectors $\mathbf{r}_{1}$ and $\mathbf{r}_{2}$ ). Thus the model is applicable for all values of $\theta_{12}$.

(3) The motion along $R$ is treated in the semiclassical approximation that leads to the replacement of the second order derivative over $\mathrm{R}$ by the first order derivative in the Schrodinger equation. This allows us to recast the problem in the form of the non-stationary Schrodinger equation with the effective time variable $t$ simply related with $R$.

(4) The states of two-electron continuum with various fixed values of the total orbital momentum $\mathrm{L}$ are considered. Two cases are particularly important: (i) ${ }^{1} \mathrm{~S}^{\mathrm{e}}$-state $(\mathrm{L}=0)$ which is the simplest feasible case 
and can be populated, e.g., by (e, 2e) process; (ii) ${ }^{1}{ }^{\text {Po }}$ state $(\mathrm{L}=1$ ) which is the only state populated

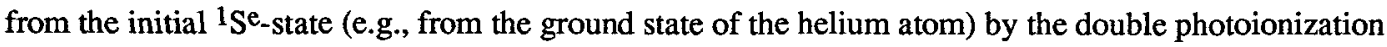
process $(\gamma, 2 \mathrm{e})$.

(5) The realistic boundary (or initial - in terms of the effective time t) conditions are formulated for the wave function at the border of the inner (reaction) zone $\mathrm{R}=\mathrm{R}_{0}$ (or $\mathrm{t}=\mathrm{t}_{0}$ ).

(6) The propagation of the wave packet from $R_{0}$ (or $t_{0}$ ) via the Coulomb zone to the domain of free electron motion is calculated numerically using the grid (Cranck-Nicholson) method.

The Sections 2 - 3 contain new results of our calculations within the extended Wannier model. We consider the time (or $\mathrm{R}$ ) evolution of the $\theta_{12}$-correlation pattern for various parameters of the system. The special attention is devoted to the integral characteristic of the angular distributions - the angular asymmetry parameter $\beta$.

\section{Angular correlation pattern for S-states of two-electron continuum.}

The basic equation of the extended Wannier ridge model (i.e. nonstationary Schrodinger equation) in this case has the form [5]:

$$
\begin{gathered}
i \frac{\partial \Phi}{\partial t}=h(R(t)) \Phi \\
Z_{0}\left(\theta_{12}\right)=2^{3 / 2} Z-\left(1-\cos \theta_{12}\right)^{-1 / 2} ; Z_{0}=2^{3 / 2}\left(Z-\frac{1}{4}\right) \\
h=-\frac{1}{2 \sin \theta_{12}} \frac{\partial}{\partial \theta_{12}} \sin \theta_{12} \frac{\partial}{\partial \theta_{12}}-\frac{1}{8}-\frac{1}{4} R(t)\left(Z_{0}\left(\theta_{12}\right)-Z_{0}\right) \\
\text { where } \quad Z_{0}\left(\theta_{12}\right)=2^{3 / 2} Z-\left(1-\cos \theta_{12}\right)^{-1 / 2} ; Z_{0}=2^{3 / 2}\left(Z-\frac{1}{4}\right)
\end{gathered}
$$

and $\mathrm{Z}$ is the charge of the residual atomic core. The wave function $\Phi\left(\theta_{12}, t\right)$ describes the angular correlations for the fixed value of the effective time $t$ or of the hyperradius $R$ which are connected by the differential relation:

$$
d t=\frac{4 d R}{p_{R} R^{2}}, \frac{1}{2} p_{R}^{2}-\frac{Z_{0}}{R}=E
$$

(it can be easily integrated analytically). Here $\mathrm{E}$ is the energy excess above the double ionization threshold.

$S$-states of two-electron continuum are populated for instance in the $(e, 2 e)$ process. The shape of the angular distribution was considered for this case by Rau [7] and Feagin [8] without specification of the boundary condition at $R=R_{0}$. The present authors have shown that in fact these results imply the assumption of some special boundary condition (zero-mode population for the oscillator describing motion over $\theta_{12}$ coordinate, for the details see Refs. $\left.[9,10]\right)$. In the present context we note that this boundary condition corresponds to the maximum for $\theta_{12}=\pi$ in the initial angular distribution at $R=$ $\mathrm{R}_{0}$.

Here we consider the opposite situation when initially (i.e. at $R=R_{0}$ ) the distribution over $\theta_{12}$ is uniform: $\Phi\left(\theta_{12}, R_{0}\right)=1$ (note that the normalization constant can be chosen arbitrarily for our purposes). As discussed above, the conventional Wannier model [6] is based on the quadratic approximation for the Hamiltonian over the variable $\left(\pi-\theta_{12}\right)$. In this approximation the uniform 
distribution always (i.e. or all $R$ ) remains uniform (this result follows straightforwardly from the general formula (14) of Ref. [10]). However the quadratic approxi- mation clearly breaks down in this case (since the large values of $\left(\pi-\theta_{12}\right)$ are involved). Thus all results in this section demonstrate the ability of the extended Wannier model to reproduce the angular correlations completely omitted in the conventional quadratic model.

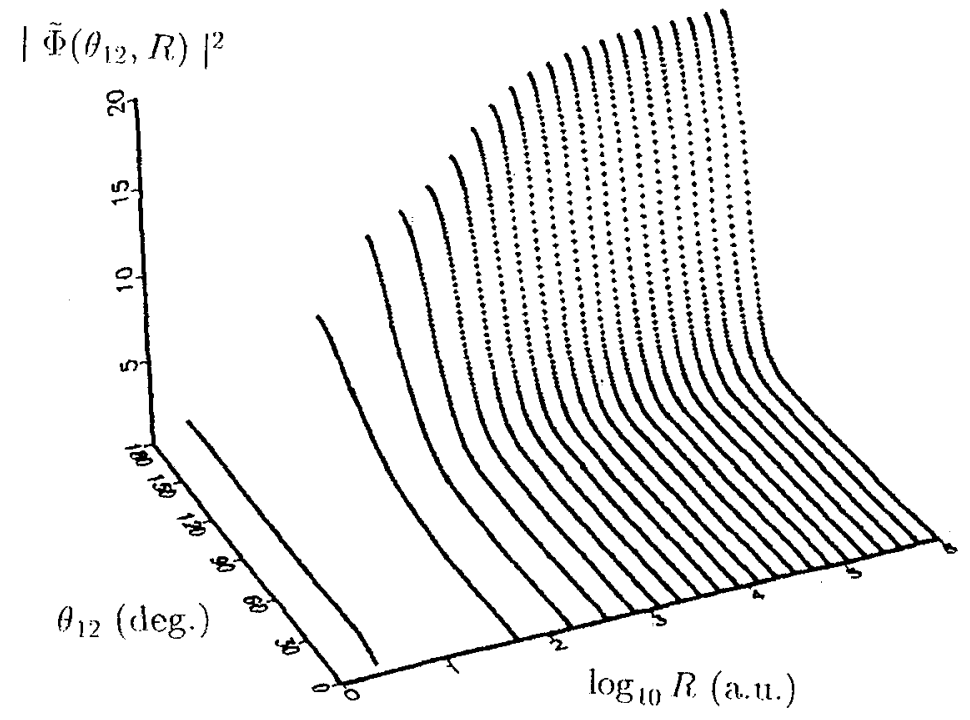

Fig.1. Evolution of the probability distribution over the correlation angle $\left|\Phi\left(\theta_{12}, R\right)\right|^{2}$ as the electrons recede from the nucleus of the helium atom $(Z=2)$ (i.e. as the hyperradius $R$ increases). The electrons are in $1 S^{e}$ continuum state, the initial distribution is uniform: $\Phi\left(\theta_{12}, R_{0}\right)=1 ; R_{0}=2 a_{0}$. The energy excess above the double ionisation threshold is $E=0.2 \mathrm{eV}$. Note the logarithmic scale over $R$.

The fig. 1 shows how the profile of the relative angular distribution evolves when $R$ increases $(Z=2$, $\mathrm{E}=0.2 \mathrm{eV}$ ). The distribution is completely uniform at $\mathrm{R}=\mathrm{R}_{0}=2 \mathrm{a}_{0}$. The formation of the peak is basically completed at $R=10^{4}$ an, although some residual evolution could be traced up to $R=10^{6} a_{0}$. The interval of $R$ essential for the profile formation increases as the energy excess $E$ decreases (see also below). The peak is centered at $\theta_{12}=\pi$ in distinction to the $\mathrm{P}^{\mathrm{O}}$ states of two-electron continuum where the peak is 'repelled' from the edge $\theta_{12}=\pi$ due to the effective centrifugal barrier (see Ref. [5] and the next section).

The situation for $Z=4$ is of some principle interest since Rau [7] had predicted the absence of the angular correlations for $Z>2$, (i.e. the uniform distributions over $\theta_{12}$ ) independent of the boundary condition. The fig. 2 clearly shows that this prediction is completely invalid even in the case of the uniform initial condition. The well manifested angular correlations exist although they look more complicated than a bell-like (or a Gaussian-like) pattern on the fig.1. The evolution of the profile with $\mathrm{R}$ 
exhibits some modulations ('wavelets'). Theirs traces persist in the final pattern. Note slight increase in the correlation function as $\theta_{12}$ deviates from $\pi$ for the larger $R$.

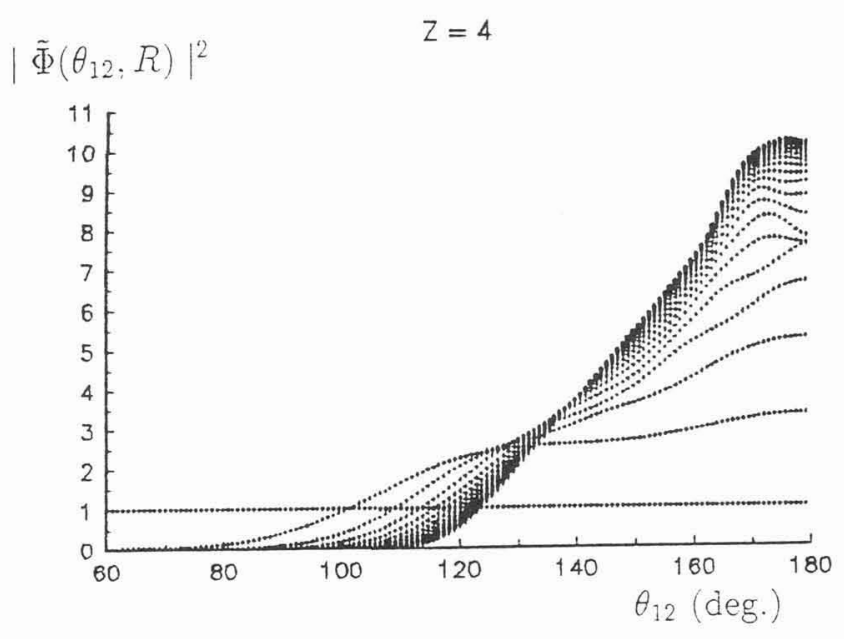

Fig.2 The same as on fig.1, but the family of the distribution functions shown on unique plane. Here $\mathrm{Z}=4, \mathrm{E}=0.0267 \mathrm{eV}$.

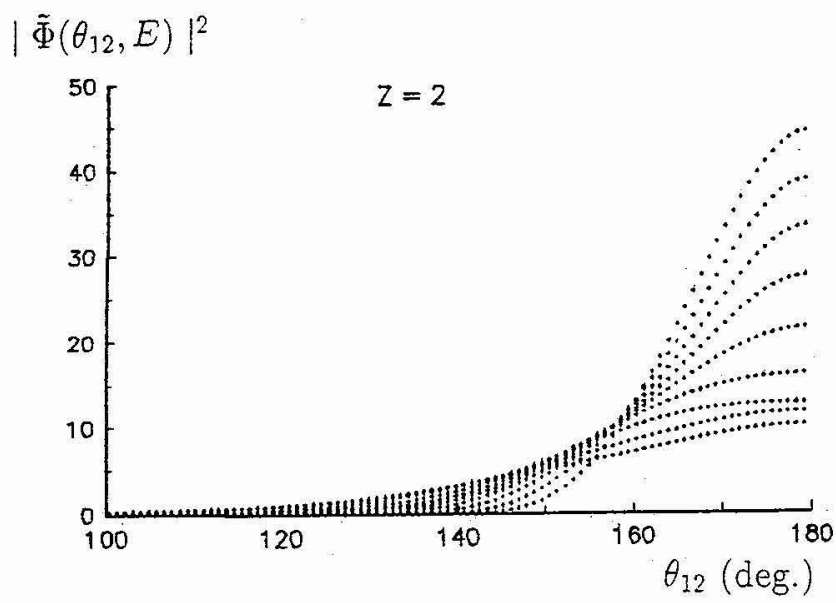

Fig. 3. The final distribution $\mid \Phi\left(\theta_{12}, E\right)^{\mid 2}$ for $Z=2$ and various values of the energy $E$. The curve with the less pronounced peak corresponds to $\mathrm{E}=1,5 \mathrm{eV}$; for the next curves the energy decreases by the factor $10^{-1 / 4}$ each time (i.e. the steps in energy are equidistant in Log E-scale).

Now we consider how the final correlation pattern (in practice calculated at $R=R_{f}=10^{6} a_{0}$ ) depends on the energy excess $\mathrm{E}$. The general tendency is quite evident qualitatively: the peak becomes more pronounced for the smaller values of $E$. In the case $Z=2$ (fig.3) the shape of peak evolves 
smoothly with $\mathrm{E}$; for the largest energies considered $(\sim 1 \mathrm{eV})$ it does not resemble a Gaussian profile. The case $Z=4$ (fig.4) looks much more intricate. For the largest values of $E$ the plateau is manifested between $130 \mathrm{deg}$ and $180 \mathrm{deg}$. For the smaller values of $\mathrm{E}$ the modulations discussed above can be noticed. For $\mathrm{E}$ equal several tens of meV the maximum of the distribution occurs at $\theta_{12} \sim 170 \mathrm{deg}$.

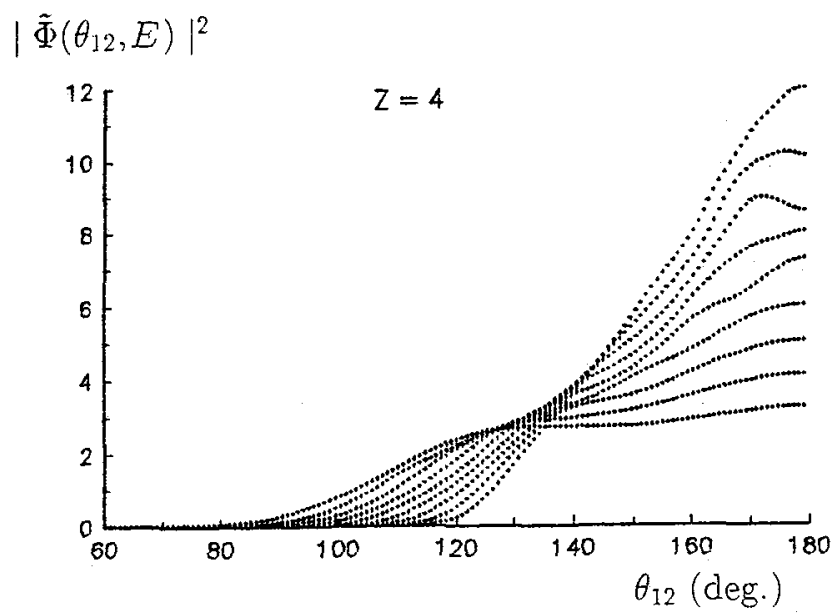

Fig. 4. The same as on Fig.3, but for $Z=4$.

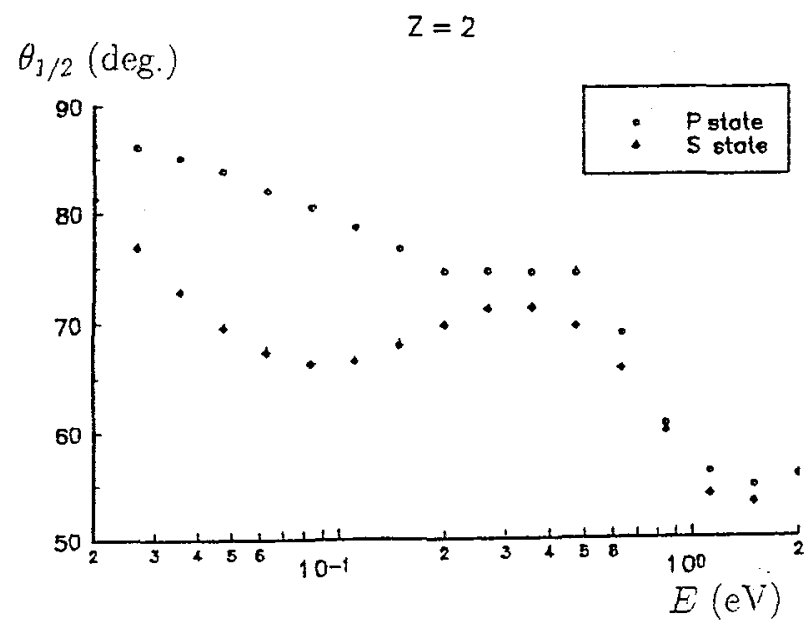

Fig. 5.The width $\theta_{12}$ of the peak in the final distribution over $\theta_{12}$ as a function of the energy $E$ for ${ }^{1} S^{2}$ qnd ${ }^{1 P 0}$ states of two-electron continuum $(Z=2)$. Nope that the peak is centered at $\theta_{12}=\pi$ for ${ }^{1} \mathrm{~S}^{\mathrm{e}_{-}}$states and shifted to the smaller $\theta_{12}$ for ${ }^{1} \mathrm{po}^{\mathrm{O}}$-states. The boundary conditions are specified in the text. 
As some overall characteristic of the distribution one can consider the full width at half maximum $\theta_{1 / 2}$. Fig.5 shows that for the situation considered in the present section this parameter varies with $E$ non-monotonically.

\section{Angular asymmetry parameter for $1 \mathrm{Po}^{\mathrm{s}}$-states.}

For the double photoionization into the $1 \mathrm{P}^{0}$ continuum the angular distributions are described by the wave function $\phi^{+}{ }_{11}\left(\theta_{12}, t\right)$ which is analogous to $\Phi\left(\theta_{12}, t\right)$ in the section 2 . Within the extended Wannier model it obeys a non-stationary Schrodinger equation of the form (2.1) but with the Hamiltonian containing the additional term $\left(1-\cos \theta_{12}\right) /\left(4 \sin ^{2} \theta_{12}\right)+\frac{1}{8}$ (see the formulas (B.3), (3.6) from Ref. [5]). This term implies the presence of the effective centrifugal barrier which inhibits penetration of the vicinity of the point $\theta_{12}=\pi$.

The boundary condition corresponding to $(\gamma, 2 \mathrm{e})$ process (in the case of uncorrelated initial $\left(\mathrm{s}^{2}\right)$ 1S state) is [5]:

$$
\phi^{+} 11\left(\theta_{12}, R_{0}\right)=\cos \left(\theta_{12} / 2\right)
$$

Due to the centrifugal barrier the peak in the final distribution is 'repelled' from the point $\theta_{12}=$ $\pi$. Its width $\theta_{1 / 2}$ is a non-monotonic function of the excess energy $E$ (fig.5) - just as in the case of the section 2.

Here we consider in more detail the integral characteristic of the angular distribution - the angular asymmetry parameter $\beta$ defined as

$$
\begin{aligned}
& \beta(R)=\frac{1}{2}+\frac{3}{2}<\cos \theta_{12}> \\
& <\cos \theta_{12}>=\frac{1}{N} \int_{0}^{\pi}\left(\phi^{+}{ }_{11}\left(\theta_{12}\right)\right)^{2} \cos \theta_{12} \sin \theta_{12} d \theta_{12} \\
& N=\int_{0}^{\pi}\left(\phi^{+}{ }_{11}\left(\theta_{12}\right)\right)^{2} \sin \theta_{12} d \theta_{12}
\end{aligned}
$$

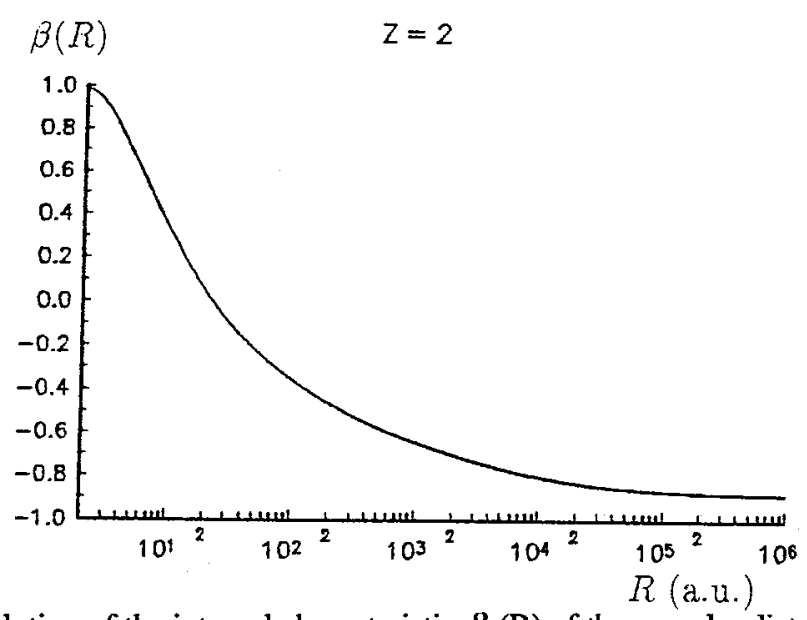

Fig.6. Evolution of the integral characteristic $\beta(R)$ of the angular distribution in 1 po continuum state with $R$ for $Z=2, E=0,0063 \mathrm{eV}$. 
The parameter $\beta(R)$ (3.2) in the limit $R \rightarrow \infty$ coincides with the asymmetry parameter $\beta$ measurable in $(\gamma, 2 \mathrm{e})$ experiments where the direction of only one ejected electron is detected [10]. The comparison with the experiment for the double photoionization of the helium atom $(Z=2)$ is presented in Ref.[5]. Here we discuss the variation of $\beta$ with the parameters of the system. It could be checked directly that for the boundary condition (3.1) one has $\beta\left(R_{0}\right)=1$.

Fig. 6 shows how $\beta(R)$ evolves with $R$ increasing, changing its sign and approaching quite slowly its limit as $R \rightarrow \infty$. The final asymmetry $\beta$ is shown on fig. 7 as a function of the excess energy $E$. The limit $\beta \rightarrow-1$ as $\mathrm{E} \rightarrow 0$ was established by Greene and Rau $[12,13]$. However these authors were unable to trace how this limit is reached with variation of $E$. This makes the result quite formal concerning its comparison with the experimental data.

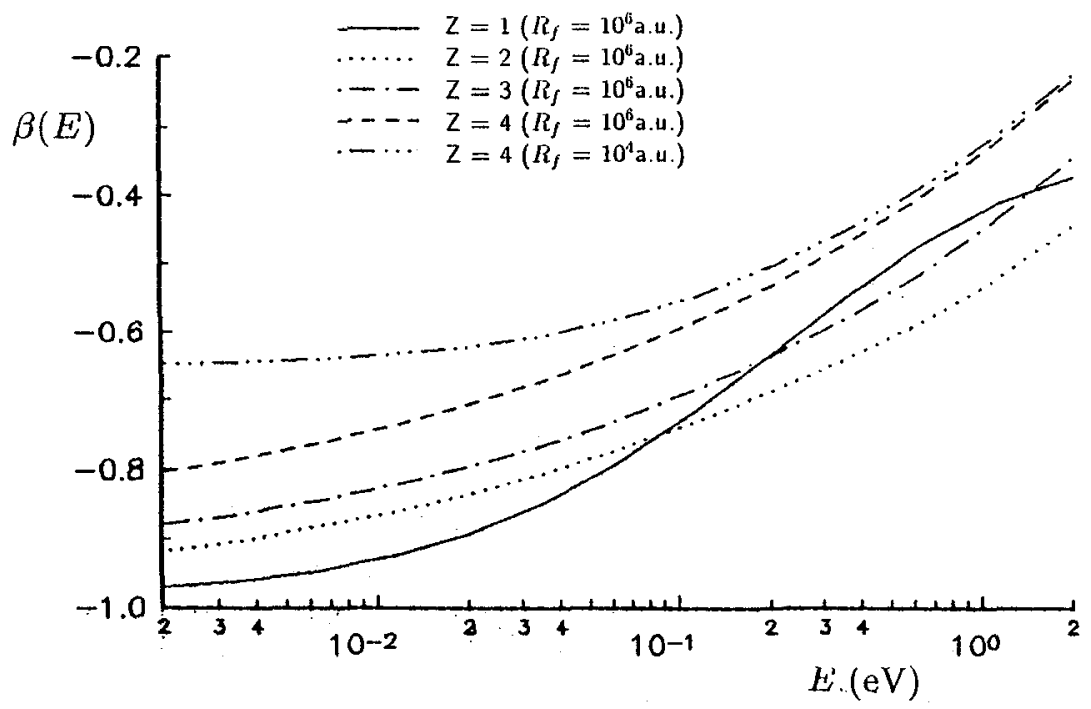

Fig.7. The asymetry parameter $\beta$ for the double ionization of helium atom $(Z=2)$ or isoelectronic ions $(Z=1,3,4)$ as a function of the excess energy $E$. The wave packet propagation is terminated at $\mathbf{R}_{f}$ $=10^{6}$ a.u.; for $Z=4$ the results for $R_{f}=10^{4}$ a.u. are also shown for comparison.

Our calculations show that the stated limit is reached quite slow ly as $\mathrm{E} \rightarrow 0$. Additionally the novel important physical feature is revealed: the substantial dependence of $\beta$ on the value of the hyperradius $\mathbf{R}_{\mathbf{f}}$ where the wave packet propagation is terminated (compare the results for various $\mathbf{R}_{\mathbf{f}}$ on the fig.7 and in the table 1). The range of integration necessary to achieve the 'theoretical value' $\beta(R \rightarrow \infty)$ increases drastically when the excess energy $E$ decreases. 
Table. Assymetry parameter $\beta$ for the double photoionisation of the helium atom $(Z=2)$. The effect of the initial $\left(R_{0}\right)$ and final $\left(R_{f}\right)$ values of the hyperradius is demonstrated.

$R_{\mathrm{f}}=10^{4}$ a.u.

$\begin{array}{cccc}\mathrm{E}, \mathrm{eV} & \mathrm{R}_{0}=2 \mathrm{a} . \mathrm{u} . & \mathrm{R}_{0}=5 \mathrm{a} . \mathrm{u} . & \mathrm{R}_{0}=12 \mathrm{a} . \mathrm{u} . \\ 2.0000 & -0.44271 & -0.37345 & -0.13757 \\ 1.1247 & -0.51757 & -0.49643 & -0.31680 \\ 0.63246 & -0.57939 & 0.59142 & -0.46321 \\ 0.35566 & -0.63125 & -0.66379 & -0.57723 \\ 0.20000 & -0.67517 & -0.71879 & -0.66280 \\ 0.11247 & -0.71208 & -0.76040 & -0.72531 \\ 0.063246 & -0.74259 & -0.79169 & -0.77053\end{array}$

$\mathbf{R}_{\mathrm{f}}=10^{6} \mathrm{a} . \mathbf{u}$

E, eV $\quad \beta \quad R_{0}=2$ a.u. $\quad R_{0}=5$ a.u. $\quad R_{0}=12$ a.u.

$\begin{array}{cccc}2.0000 & -0.44584 & -0.37956 & -0.14655 \\ 1.1247 & -0.52159 & -0.50415 & -0.32852 \\ 0.63246 & -0.58531 & -0.60131 & -0.47788 \\ 0.35566 & -0.64029 & -0.67649 & -0.59515 \\ 0.20000 & -0.68866 & -0.73496 & -0.68479 \\ 0.11247 & -0.73174 & -0.78104 & -0.75248 \\ 0.063246 & -0.77023 & -0.81797 & -0.80354\end{array}$

In a real experimental situation the effective value of $R_{f}$ is determined by such factors as the density of atoms in the device and the presence of the stray electric fields in it. As $R$ increases, the potential of the electron-electron interaction becomes smaller than the aforementioned additional interactions.(The realistic experiments imply also that the final energies of the electrons are fixed (usually the condition of the equal sharing of the energy $\mathrm{E}$ between the electrons is assumed). The precision $\Delta \mathrm{E}$ with which this condition is controlled introduces an additional characteristic energy parameter in the problem.) The propagation of the wave packet beyond this distance is physically senseless.

The accurate analyses of the mentioned factors poses a serious problem which is beyound the scope of the present paper. However we would like to stress here the general conclusion of principle importance: Any statement of the theory which is not valid for a reasonable value of $\mathrm{R}_{\mathrm{f}}$ hardly could be observed in the experiment.. Note that the similar situation was revealed by the authors [14] for the energy dependence of the double electron escape cross sections.

The vital condition for the applicability of the Wannier theory is that its result should exhibit only weak a dependence on the position of the border $R=R_{0}$ where the boundary condition is imposed. This point was also never studied before. The situation for the extended Wannier model is characterized by table 1 where we show how the choice of $R_{0}$ influences the final asymmetry parameter $\beta$. The general conclusion is that some dependence on $\mathrm{R}_{0}$ persists, but it seems to be tolerable for a reasonable choice of 
$R_{0}$. One of the values shown in the table $\left(R_{0}=12\right.$ a.u.) is unphysically large and the related value of $\beta$ is substantially different for the highest values of the energy $E$ considered. For the smaller values of $E$ the influence of the choice of $R_{0}$ becomes weaker in agreement with the spirit of the Wannier approach. Nevertheless the refined treatment of the inner region would be one of the directions for future researches.

\section{Conclusion .}

The results presented above demonstrate the capacity of the extended Wannier model. In the forthcoming publications it will be applied to the calculation of the triple differential cross sections and comparison with experiment $[2,3]$. The other directions of the developments could be indicated: (i) application of the model to various symmetries of the initial and final state; (ii) more exact formulation of the boundary conditions with account taken of the specifics of the initial state including electron correlations; (iii) calculation of the absolute values for the cross sections of the double escape.

This work was supported by the grant No 93-02-14888 from the Russian Foundation for Fundamental Researches. It was completed during the visit of one of the authors (VNO) to The University of Bielefeld supported by the Deutsche Forschungsgemeinschaft in Sonderforschungbereich 216 "Polarisation und Korrelation in atomaren Stoßprozessen".

\section{References}

1. Mazeau J., Selles P., Waymel D. and Huetz A., Phys.Rev.Lett. 67 (1991) 820.

2. Waymel D., Andric L., Mazeau J., Selles P. and Huetz A., J.Phys.B: At.Mol.Opt.Phys. 26 (1993) L123.

3. Schwarzkopf O., Krassig B., Elmiger J. and Schmidt V., Phys.Rev..Lett. 70 (1993) 3008.

4. Wannier G.H., Phys.Rev. 90 (1953) 817.

5. Kazansky A.K. and Ostrovsky V.N., J.Phys.B: At.Mol.Opt.Phys. (submitted for publication); XVIII Int.Conf. on the Physics of Electrolnic and Atomic Collisions, Abstracts of Papers, p.3.

6. Read F.H., Electron Impact Ionization $\backslash$ Ed. T.D.Mark and G.H.Dunn, 1985 (New York: Springer) pp 42-86.

7. Rau A.R.P., J.Phys.B: At. Mol. Phys. 9 (1976) L283.

8. Feagin J.M., J.Phys.B: At. Mol. Phys. 17 (1984) 2433.

9. Kazansky A.K. and Ostrovsky V.N., J.Phys.B: At.Mol.Opt.Phys. 26 (1993) 2231.

10. Kazansky A.K. and Ostrovsky V.N., Phys.Rev.A 48 (1993) R871.

11. Hall R.I., McConkey A.G., Avaldi L., Ellis K., MacDonald M.A., Dawber G. and King G.C., J.Phys.B:At.Mol.Opt.Phys. 25 (1992) 1195.

12. Greene C.H. and Rau A.R.P., J.Phys.B: At.Mol. Phys. 16 (1983) 99.

13. Greene C.H., J.Phys.B: At. Mol. Phys. 20 (1987) L357.

14. Kazansky A.K. and Ostrovsky V.N., J.Phys.B: At. Mol. Opt. Phys. 25 (1992) 2121 